\title{
A Review on the Solutions of Difference Equations via Integer Sequences such as Fibonacci Numbers and Tribonacci Numbers
}

\author{
İnci Okumuş ${ }^{1 *}$, Yüksel Soykan²
}

\begin{abstract}
In this review article, we study the recent investigations on the forms of solutions of systems difference equations and difference equations in terms of well-known integer sequences such as Fibonacci numbers, Padovan numbers. We focus on the papers given some interesting relationships both between the exact solutions of difference equations and the integer sequences and between the equilibrium points of difference equations and golden ratio.
\end{abstract}

Keywords: Difference equations, Equilibrium point, Fibonacci number, Solutions, Tribonacci numbers 2010 AMS: 39A10, 39A30

${ }^{1}$ Department of Mathematics, Zonguldak Bülent Ecevit University, Zonguldak, Turkey, ORCID: 0000-0003-3711-8144 ${ }^{2}$ Department of Mathematics, Zonguldak Bülent Ecevit University, Zonguldak, Turkey, ORCID: 0000-0002-1895-211X *Corresponding author: inci_okumus_90@hotmail.com

Received: 15 July 2019, Accepted: 01 October 2019, Available online: 29 December 2019

\section{Introduction}

Difference equations and systems of difference equations are great importance in the field of mathematics as well as in other sciences. The applications of the theory of difference equations appear as discrete mathematical models of many phenomena such as in biology, economics, ecology, control theory, physics, engineering, population dynamics and so forth. Recently, there has been a growing interest in the study of finding closed-form solutions of difference equations and systems of difference equations. Some of the forms of solutions of these equations are representable via well-known integer sequences such as Fibonacci numbers, Lucas numbers, Pell numbers and Padovan numbers.

Now, we give information about integer sequences that establish a large part of our study.

- The Fibonacci sequence is defined by

$$
F_{n}=F_{n-1}+F_{n-2}, \quad n \geq 2
$$

with initial conditions $F_{0}=0, F_{1}=1$. Also, it is obtained to extend the Fibonacci sequence backward as

$$
F_{-n}=(-1)^{n+1} F_{n} .
$$

The characteristic equation of (1.1) is $x^{2}-x-1=0$ such that the roots

$$
\alpha=\frac{1+\sqrt{5}}{2} \text { (golden ratio) and } \beta=\frac{1-\sqrt{5}}{2} \text {. }
$$


Also, there exists the following limit

$$
\lim _{n \rightarrow \infty} \frac{F_{n+1}}{F_{n}}=\alpha,
$$

where $F_{n}$ is $n$th Fibonacci number.

- The Padovan sequence is defined by

$$
P_{n}=P_{n-2}+P_{n-3}, \quad n \in \mathbb{N}
$$

with initial conditions $P_{-2}=0, P_{-1}=0, P_{0}=1$.

The characteristic equation of (1.2) is $x^{3}-x-1=0$ such that the roots

$$
\begin{aligned}
p & =\frac{r^{2}+12}{6 r} \\
q & =-\frac{r^{2}+12}{12 r}-i \frac{\sqrt{3}}{2}\left(\frac{r}{6}-\frac{2}{r}\right) \\
t & =-\frac{r^{2}+12}{12 r}+i \frac{\sqrt{3}}{2}\left(\frac{r}{6}-\frac{2}{r}\right)
\end{aligned}
$$

where $r=\sqrt[3]{108+12 \sqrt{69}}$ and the unique real root is $p$ named as plastic number. Also, there exists the following limit

$$
\lim _{n \rightarrow \infty} \frac{P_{n+1}}{P_{n}}=p
$$

where $P_{n}$ is $n$th Padovan number.

- Horadam sequence, a generalization of Fibonacci sequence, $\left(W_{n}(a, b ; p, q)\right)_{n \geq 0}$ or simply $\left(W_{n}\right)_{n \geq 0}$ is defined by

$$
W_{n}=p W_{n-1}+q W_{n-2}, \quad W_{0}=a, W_{1}=b, \quad n \geq 2,
$$

where $a, b, p$ and $q$ are arbitrary real numbers.

The characteristic equation of (1.3) is $x^{2}-p x-q=0$ such that the roots

$$
\lambda=\frac{p+\sqrt{p^{2}+4 q}}{2} \text { and } \mu=\frac{p-\sqrt{p^{2}+4 q}}{2} .
$$

Also, there exists the following limit

$$
\lim _{n \rightarrow \infty} \frac{W_{n+1}}{W_{n}}=\lambda,
$$

where $W_{n}$ is $n$th Horadam number.

- The generalized Padovan sequence, an extension of the padovan sequence, is defined by

$$
S_{n}=p S_{n-2}+q S_{n-3}, \quad n \in \mathbb{N}
$$

with initial conditions $S_{-2}=0, S_{-1}=0, S_{0}=1$, where $p$ and $q$ are arbitrary real numbers.

The characteristic equation of (1.4) is $x^{3}-p x-q=0$ such that the roots

$$
\begin{aligned}
\phi & =\frac{R^{2}+12 p}{6 R} \\
\varphi & =-\frac{R^{2}+12 p}{12 R}+i \frac{\sqrt{3}}{2}\left(\frac{R}{6}-\frac{2 p}{R}\right) \\
\psi & =-\frac{R^{2}+12 p}{12 R}-i \frac{\sqrt{3}}{2}\left(\frac{R}{6}-\frac{2 p}{R}\right)
\end{aligned}
$$


where $R=\sqrt[3]{108 q+12 \sqrt{-12 p^{3}+81 q^{2}}}$. Also, there exists the following limit

$$
\lim _{n \rightarrow \infty} \frac{S_{n+1}}{S_{n}}=\phi
$$

where $S_{n}$ is $n$th generalized Padovan number.

- Generalized Tribonacci sequence is defined by

$$
V_{n}=r V_{n-1}+s V_{n-2}+t V_{n-3}, \quad n \geq 3
$$

with initial conditions $V_{0}=a, V_{1}=b, V_{2}=c$ and $r, s, t$ are real numbers.

The characteristic equation is $x^{3}-r x^{2}-s x-t=0$, whose roots are

$$
\begin{aligned}
& \alpha=\alpha(r, s, t)=\frac{r}{3}+A+B \\
& \beta=\beta(r, s, t)=\frac{r}{3}+\omega A+\omega^{2} B \\
& \gamma=\gamma(r, s, t)=\frac{r}{3}+\omega^{2} A+\omega B
\end{aligned}
$$

where

$$
\begin{aligned}
& A=\left(\frac{r^{3}}{27}+\frac{r s}{6}+\frac{t}{2}+\sqrt{\Delta}\right)^{1 / 3}, B=\left(\frac{r^{3}}{27}+\frac{r s}{6}+\frac{t}{2}-\sqrt{\Delta}\right)^{1 / 3} \\
& \Delta=\Delta(r, s, t)=\frac{r^{3} t}{27}-\frac{r^{2} s^{2}}{108}+\frac{r s t}{6}-\frac{s^{3}}{27}+\frac{t^{2}}{4} \\
& \omega=\frac{-1+i \sqrt{3}}{2}=\exp (2 \pi i / 3) .
\end{aligned}
$$

- When $r=1, s=1, t=1$ and $a=0, b=1, c=1$ in (1.5), Tribonacci sequence is defined by

$$
T_{n+3}=T_{n+2}+T_{n+1}+T_{n},
$$

with initial conditions $T_{0}=0, T_{1}=1, T_{2}=1$. Also, it can be extended the Tribonacci sequence backward (negative subscripts) as

$$
T_{-n}=T_{-n+3}-T_{-n+2}-T_{-n+1} .
$$

The characteristic equation of (1.6) is

$$
x^{3}-x^{2}-x-1=0
$$

such that the roots

$$
\begin{aligned}
& \alpha=\frac{1+\sqrt[3]{19+3 \sqrt{33}}+\sqrt[3]{19-3 \sqrt{33}}}{3} \\
& \beta=\frac{1+\omega \sqrt[3]{19+3 \sqrt{33}}+\omega^{2} \sqrt[3]{19-3 \sqrt{33}}}{3} \\
& \gamma=\frac{1+\omega^{2} \sqrt[3]{19+3 \sqrt{33}}+\omega \sqrt[3]{19-3 \sqrt{33}}}{3}
\end{aligned}
$$

where $\alpha$ is called Tribonacci constant and

$$
\omega=\frac{-1+i \sqrt{3}}{2}=\exp (2 \pi i / 3)
$$

is a primitive cube root of unity.

Furthermore, there exist the following limit

$$
\lim _{n \rightarrow \infty} \frac{T_{n+r}}{T_{n}}=\alpha^{r},
$$

where $r \in \mathbb{Z}$ and $T_{n}$ is the $n$th Tribonacci number. 
- Lucas sequence is defined by

$$
L_{n}=L_{n-1}+L_{n-2}, \quad L_{0}=2, L_{1}=1 .
$$

- Pell sequence is defined by

$$
P_{n}=2 P_{n-1}+P_{n-2}, \quad P_{0}=0, P_{1}=1 .
$$

- Pell-Lucas sequence is defined by

$$
P_{n}=2 P_{n-1}+P_{n-2}, \quad P_{0}=2, P_{1}=2 .
$$

- Jacobsthal sequence is defined by

$$
J_{n}=J_{n-1}+2 J_{n-2}, \quad J_{0}=0, J_{1}=1 .
$$

- Jacobsthal-Lucas sequence is defined by

$$
J_{n}=J_{n-1}+2 J_{n-2}, \quad J_{0}=2, J_{1}=1 .
$$

- Perrin sequence is defined by

$$
P_{n}=P_{n-2}+P_{n-3}, \quad P_{0}=3, P_{1}=0, P_{2}=2 .
$$

\section{Literature review}

In [1], Tollu et al. considered the following difference equations

$$
x_{n+1}=\frac{1}{1+x_{n}}, \quad y_{n+1}=\frac{1}{-1+y_{n}}, \quad n=0,1, \ldots,
$$

such that their solutions are associated with Fibonacci numbers, where initial conditions are $x_{0} \in \mathbb{R}-\left\{-\frac{F_{m+1}}{F_{m}}\right\}_{m=1}^{\infty}$ and $y_{0} \in \mathbb{R}-\left\{-\frac{F_{m+1}}{F_{m}}\right\}_{m=1}^{\infty}$ and $F_{m}$ is the $m$ th Fibonacci number.

They investigated the some relationships both between Fibonacci numbers and solutions of equations (2.1) and between the golden ratio and equilibrium points of equations (2.1). Then, they proved that: the solutions of equations (2.1) are given by

$$
x_{n}=\frac{F_{n}+F_{n-1} x_{0}}{F_{n+1}+F_{n} x_{0}}, y_{n}=\frac{F_{-n}+F_{-(n-1)} y_{0}}{F_{-(n+1)}+F_{-n} y_{0}},
$$

where $F_{n}$ is the $n$th Fibonacci number, and the nontrival solutions of equations (2.1) converge to $-\beta$ and $\beta$, so that $\beta$ is conjugate to the golden ratio.

Next, Rabago [2] presented a theoretical explanation in deriving the closed-form solution of Eq. (2.1) which Tollu et al. studied in [1] and provided another approach in proving Sroysang's conjecture (2013).

Then, in [3], Yazlik et al. studied the following rational difference equation systems

$$
x_{n+1}=\frac{x_{n-1} \pm 1}{y_{n} x_{n-1}}, \quad y_{n+1}=\frac{y_{n-1} \pm 1}{x_{n} y_{n-1}}, n=0,1, \ldots,
$$

such that their solutions associated with Padovan numbers. In their study, they obtained that the forms of solutions of system (2.2) are as follows

$$
\begin{aligned}
& x_{n}= \begin{cases}\mp \frac{P_{n} x_{-1} y_{0} \mp P_{n+1} x_{-1}+P_{n-1}}{P_{n-1} x_{-1} y_{0} \mp P_{n} x_{-1}+P_{n-2},} & \text { if } n \text { is odd } \\
\mp \frac{P_{n} y_{-1} x_{0} \mp P_{n+1} y_{-1}+P_{n-1}}{P_{n-1} y_{-1} x_{0} \mp P_{n} y_{-1}+P_{n-2}}, & \text { if } n \text { is even }\end{cases} \\
& y_{n}= \begin{cases}\mp \frac{P_{n} y_{-1} x_{0} \mp P_{n+1} y_{-1}+P_{n-1}}{P_{n-1} y_{-1} x_{0} \mp P_{n} y_{-1}+P_{n-2},} & \text { if } n \text { is odd } \\
\mp \frac{P_{n} x_{-1} y_{0} \mp P_{n+1} x_{-1}+P_{n-1}}{P_{n-1} x_{-1} y_{0} \mp P_{n} x_{-1}+P_{n-2}}, & \text { if } n \text { is even }\end{cases}
\end{aligned}
$$


where $P_{n}$ is the $n$th Padovan number. Also, they demonstrated that every solutions of the systems (2.2) converge to point $(p, p)$ and $(-p,-p)$, where $p$ is the plastic number.

Tollu et al. [4] considered the following four Riccati difference equations

$$
x_{n+1}=\frac{1+x_{n}}{x_{n}}, y_{n+1}=\frac{1-y_{n}}{y_{n}}, u_{n+1}=\frac{1}{u_{n}+1}, v_{n+1}=\frac{1}{v_{n}-1},
$$

in which the initial conditions are real numbers. They derived the formulae for the solutions of equations (2.3) are given by

$$
\begin{aligned}
x_{n} & =\frac{F_{n+1} x_{0}+F_{n}}{F_{n} x_{0}+F_{n-1}}, \\
y_{n} & =\frac{F_{-(n+1)} y_{0}+F_{-n}}{F_{-n} y_{0}+F_{-(n-1)}}, \\
u_{n} & =\frac{F_{n}+F_{n-1} u_{0}}{F_{n+1}+F_{n} u_{0}}, \\
v_{n} & =\frac{F_{-n}+F_{-(n-1)} v_{0}}{F_{-(n+1)}+F_{-n} v_{0}},
\end{aligned}
$$

where $F_{n}$ is $n$th Fibonacci number, $F_{-n}$ is $n$th negative Fibonacci number. In addition to, they stated the asymptotic behaviors of the solutions of these equations and introduced that every solutions of these equations converge to their positive or negative equilibrium points.

Also, they in [5] studied the systems of difference equations

$$
x_{n+1}=\frac{1+p_{n}}{q_{n}}, y_{n+1}=\frac{1+r_{n}}{s_{n}}, n \in \mathbb{N}_{0},
$$

where each of the sequences $p_{n}, q_{n}, r_{n}$ and $s_{n}$ is some of the sequences $x_{n}$ or $y_{n}$ by their own. They solved fourteen systems out of sixteen possible systems. In particularly, the representation formulae of solutions of twelve systems were stated via Fibonacci numbers. Also, for ten systems, they expressed that the solutions of these systems tend to the unique point $(\alpha, \alpha)$ where $\alpha$ is the golden ratio.

In [6], Halim concerned with the following systems of rational difference equations

$$
x_{n+1}=\frac{1}{1+y_{n}}, y_{n+1}=\frac{1}{1+x_{n}}, n=0,1, \ldots
$$

and

$$
x_{n+1}=\frac{1}{1-y_{n}}, \quad y_{n+1}=\frac{1}{1-x_{n}}, \quad n=0,1, \ldots,
$$

initial conditions are arbitrary nonzero real numbers. He determined the form of solutions of system (2.4) as given below

$$
\begin{array}{ll}
x_{2 n-1}=\frac{F_{2 n-1}+F_{2 n-2} y_{0}}{F_{2 n}+F_{2 n-1} y_{0}}, & x_{2 n}=\frac{F_{2 n}+F_{2 n-1} x_{0}}{F_{2 n+1}+F_{2 n} x_{0}}, \\
y_{2 n-1}=\frac{F_{2 n-1}+F_{2 n-2} x_{0}}{F_{2 n}+F_{2 n-1} x_{0}}, & y_{2 n}=\frac{F_{2 n}+F_{2 n-1} y_{0}}{F_{2 n+1}+F_{2 n} y_{0}},
\end{array}
$$

and proved that the equilibrium point $E$ of system (2.4) is globally asymptotically stable, where $E=\left(\frac{-1+\sqrt{5}}{2}, \frac{-1+\sqrt{5}}{2}\right)=\left(\frac{1}{\alpha}, \frac{1}{\alpha}\right)$, where $\alpha$ is the golden ratio. Furthermore, he established the solutions of system (2.5) are periodic with period six and are unstable.

In [7], Bacani and Rabago studied the behavior of solutions of the following nonlinear difference equations

$$
x_{n+1}=\frac{q}{p+x_{n}^{v}} \text { and } y_{n+1}=\frac{q}{-p+y_{n}^{v}}
$$

where $p, q \in \mathbb{R}^{+}$and $v \in \mathbb{N}$. They proved that the solutions of equations (2.6) in case $v=1$ are as follows

$$
\begin{aligned}
& x_{n}=\frac{q W_{n}+x_{0} q W_{n-1}}{W_{n+1}+x_{0} W_{n}}, \\
& y_{n}=\frac{q W_{-n}+y_{0} q W_{-(n-1)}}{W_{-(n+1)}+y_{0} W_{-n}},
\end{aligned}
$$


where $W_{n}$ is the $n$th Horadam number.

In [8], Halim and Bayram investigated the solutions, stability character, and asymptotic behavior of the difference equation

$$
x_{n+1}=\frac{\alpha}{\beta+\gamma x_{n-k}}, n \in \mathbb{N}_{0},
$$

where the initial conditions $x_{-k}, x_{-k+1}, \ldots, x_{0}$ are nonzero real numbers, such that its solutions are associated to Horadam numbers, which are generalized Fibonacci numbers. Firstly, they had the difference equation

$$
x_{n+1}=\frac{q}{p+x_{n-k}},
$$

by putting $q=\frac{\alpha}{\gamma}$ and $p=\frac{\beta}{\gamma}$. Then, they proved that the forms of the solutions of difference equation (2.7) are as follows

$$
x_{(k+1) n+i}=\frac{W_{n+1}+W_{n} x_{i-(k+1)}}{W_{n+2}+W_{n+1} x_{i-(k+1)}} q, i=1,2, \ldots, k+1,
$$

where $W_{n}$ is the $n$th Horadam number. Also, they obtained that the equilibrium point $E$ of difference equation (2.7) is globally asymptotically stable, where $E=\frac{-p+\sqrt{p^{2}+4 q}}{2}$.

Then, in [9] Halim considered the system of difference equations

$$
x_{n+1}=\frac{1}{1+y_{n-2}}, \quad y_{n+1}=\frac{1}{1+x_{n-2}}, n=0,1, \ldots,
$$

where $\mathbb{N}_{0}=\mathbb{N} \cup\{0\}$ and the initial conditions $x_{-2}, x_{-1}, x_{0}, y_{-2}, y_{-1}$, and $y_{0}$ are real numbers. He presented the relationship between Fibonacci numbers and the solutions of system (2.8), i.e., the form of the solutions of system (2.8) are given by

$$
\begin{array}{ll}
x_{6 n+i}=\frac{F_{2 n+1}+F_{2 n} y_{i-3}}{F_{2 n+2}+F_{2 n+1} y_{i-3}}, & i=1,2,3, \\
y_{6 n+i}=\frac{F_{2 n+1}+F_{2 n} x_{i-3}}{F_{2 n+2}+F_{2 n+1} x_{i-3}}, & i=1,2,3, \\
x_{6 n+i}=\frac{F_{2 n+2}+F_{2 n+1} x_{i-6}}{F_{2 n+3}+F_{2 n+2} x_{i-6}}, & i=4,5,6, \\
y_{6 n+i}=\frac{F_{2 n+2}+F_{2 n+1} y_{i-6}}{F_{2 n+3}+F_{2 n+2} y_{i-6}}, & i=4,5,6,
\end{array}
$$

where $F_{n}$ is the $n$th Fibonacci number. Otherwise, he showed that the equilibrium point $E$ of system (2.8) is globally asymptotically stable, where $E=\left(\frac{-1+\sqrt{5}}{2}, \frac{-1+\sqrt{5}}{2}\right)$.

El-Dessoky in [10] dealt with the following difference equation

$$
x_{n+1}=a x_{n}+\frac{\alpha x_{n} x_{n-l}}{\beta x_{n}+\gamma x_{n-k}}, n=0,1, \ldots
$$

where the parameters $\alpha, \beta, \gamma$ and $a$ and the initial conditions $x_{-t}, x_{-t+1}, \quad, x_{-1}$ and $x_{0}$ where $t=\max \{l, k\}$ are positive real numbers. He introduced the explicit formula of solutions of some special cases of Eq. (2.9) via Fibonacci numbers and also, discussed the global behavior of solutions of Eq. (2.9).

In [11], Halim and Rabago studied the systems of difference equaions

$$
x_{n+1}=\frac{1}{ \pm 1 \pm y_{n-k}}, \quad y_{n+1}=\frac{1}{ \pm 1 \pm x_{n-k}}, n, k \in \mathbb{N}_{0},
$$

where the initial conditions $x_{-k}, x_{-k+1}, \ldots, x_{0}, y_{-k}, y_{-k+1}, \ldots, y_{0}$ are nonzero real numbers.

Initially, they examined the form and behavior of solutions of system of difference equations

$$
x_{n+1}=\frac{1}{1+y_{n-k}}, \quad y_{n+1}=\frac{1}{1+x_{n-k}} .
$$

Therefore, they determined that the exact solutions of system (2.10) are as follows

$$
\begin{aligned}
x_{2(k+1) n+i}=\frac{F_{2 n+1}+F_{2 n} y_{i-(k+1)}}{F_{2 n+2}+F_{2 n+1} y_{i-(k+1)}}, & i=1,2, \ldots, k+1, \\
y_{2(k+1) n+i}=\frac{F_{2 n+1}+F_{2 n} x_{i-(k+1)}}{F_{2 n+2}+F_{2 n+1} x_{i-(k+1)}}, & i=1,2, \ldots, k+1, \\
x_{2(k+1) n+i}=\frac{F_{2 n+2}+F_{2 n+1} x_{i-(2 k+2)}}{F_{2 n+3}+F_{2 n+2} x_{i-(2 k+2)}}, & i=k+2, \ldots, 2 k+2, \\
y_{2(k+1) n+i}=\frac{F_{2 n+2}+F_{2 n+1} y_{i-(2 k+2)}}{F_{2 n+3}+F_{2 n+2} y_{i-(2 k+2)}}, & i=k+2, \ldots, 2 k+2,
\end{aligned}
$$


and the equilibrium point of system (2.10) is globally asymptotically stable. In addition, the authors gave some results for other systems.

Then, in [12], the authors studied the rational difference equation

$$
x_{n+1}=\frac{\alpha x_{n-1}+\beta}{\gamma x_{n} x_{n-1}}, n \in \mathbb{N}_{0},
$$

where $\mathbb{N}_{0}=\mathbb{N} \cup\{0\}, \alpha, \beta, \gamma \in \mathbb{R}^{+}$and the initial conditions nonzero real numbers and also investigated the two-dimensional case of the this equation given by

$$
x_{n+1}=\frac{\alpha x_{n-1}+\beta}{\gamma y_{n} x_{n-1}}, y_{n+1}=\frac{\alpha y_{n-1}+\beta}{\gamma x_{n} y_{n-1}}, n \in \mathbb{N}_{0} .
$$

Firstly, they reduced the difference equation (2.11) to the difference equation

$$
x_{n+1}=\frac{p x_{n-1}+q}{x_{n} x_{n-1}}
$$

by using changes variables $p=\frac{\alpha}{\gamma}$ and $q=\frac{\beta}{\gamma}$. Then, they presented that the closed-form solution of difference equation (2.13) is given by

$$
x_{n}=\frac{S_{n+1} x_{-1}+S_{n} x_{0} x_{-1}+q S_{n-1}}{S_{n} x_{-1}+S_{n-1} x_{0} x_{-1}+q S_{n-2}},
$$

where $S_{n}$ is the $n$th generalized Padovan number and the equilibrium point of Eq (2.13) is globally asymptotically stable.

Later, they reduced the system of difference equation (2.12) to the system

$$
x_{n+1}=\frac{p x_{n-1}+q}{y_{n} x_{n-1}}, y_{n+1}=\frac{p y_{n-1}+q}{x_{n} y_{n-1}}
$$

by using changes variables $p=\frac{\alpha}{\gamma}$ and $q=\frac{\beta}{\gamma}$. Then, they presented that the closed-form solutions of system (2.14) are given by

$$
\begin{aligned}
& x_{n}= \begin{cases}\frac{S_{n+1} y_{-1}+S_{n} x_{0} y_{-1}+q S_{n-1}}{S_{n} y_{-1}+S_{n-1} x_{0} y_{-1}+q S_{n-2}}, & \text { if } n \text { is even, } \\
\frac{S_{n+1} x_{-1}+S_{n} y_{0} x_{-1}+q S_{n-1}}{S_{n} x_{-1}+S_{n-1} y_{0} x_{-1}+q S_{n-2}}, & \text { if } n \text { is odd, }\end{cases} \\
& y_{n}= \begin{cases}\frac{S_{n+1} x_{-1}+S_{n} y_{0} x_{-1}+q S_{n-1}}{S_{n} x_{-1}+S_{n-1} y_{0} x_{-1}+q S_{n-2}}, & \text { if } n \text { is even, } \\
\frac{S_{n+1} y_{-1}+S_{n} x_{-1}+q S_{n-1}}{S_{n} y_{-1}+S_{n-1} x_{0} y_{-1}+q S_{n-2}}, & \text { if } n \text { is odd, }\end{cases}
\end{aligned}
$$

and the equilibrium point of the system (2.14) is global attractor.

Then, in [13], Stevic et al. the following nonlinear second-order difference equation

$$
x_{n+1}=a+\frac{b}{x_{n}}+\frac{c}{x_{n} x_{n-1}}, n \in \mathbb{N}_{0},
$$

in which parameters $a, b, c$ and the initial values $x_{-1}$ and $x_{0}$ are complex numbers such that $c \neq 0$. Next, they used the following change of variables

$$
x_{n}=\frac{y_{n}}{y_{n-1}},
$$

and obtained the following third-order linear difference equation with constant coefficients

$$
y_{n+1}=a y_{n}+b y_{n-1}+c y_{n-2} \text {. }
$$

After, they introduced that the representation formula of every solution of Eq. (2.15) is

$$
x_{n}=\frac{\left(s_{n+1}-a s_{n}\right) x_{-1}+s_{n} x_{0} x_{-1}+c s_{n-1}}{\left(s_{n}-a s_{n-1}\right) x_{-1}+s_{n-1} x_{0} x_{-1}+c s_{n-2}},
$$

where $s_{n}$ is the $n$th generalized Padovan number. Note that, Eq. (2.11) is a special case of Eq. (2.15) such that $a=0$. 
Alotaibi et al. in [14] considered the following systems of difference equations

$$
x_{n+1}=\frac{y_{n} y_{n-2}}{x_{n-1}+y_{n-2}}, y_{n+1}=\frac{x_{n} x_{n-2}}{ \pm y_{n-1} \pm x_{n-2}}, n=0,1, \ldots,
$$

where the initial conditions $x_{-2}, x_{-1}, x_{0}, y_{-2}, y_{-1}, y_{0}$ are arbitrary positive real numbers. They analyzed the solutions of the systems (2.16) such that their solutions are associated with Fibonacci numbers.

In [15], El-Dessoky et al. examined the following difference equation

$$
y_{n+1}=\alpha y_{n}+\frac{\beta y_{n} y_{n-3}}{A y_{n-4}+B y_{n-3}}, n=0,1, \ldots,
$$

where $\alpha, \beta, A$, and $B$ are real numbers and the initial values $y_{-4}, y_{-3}, y_{-2}, y_{-1}$ and $y_{0}$ are positive real numbers. They presented the solutions of Eq. (2.17) in terms of Fibonacci numbers according to some special cases of the parameters $\alpha, \beta, A$, and $B$.

Then, in [16], Matsunaga and Suzuki studied the following system of rational difference equations

$$
x_{n+1}=\frac{a y_{n}+b}{c y_{n}+d}, y_{n+1}=\frac{a x_{n}+b}{c x_{n}+d}, n=0,1, \ldots,
$$

where the parameters $a, b, c, d$ and the initial values $x_{0}, y_{0}$ are real numbers. They obtained that the explicit solutions of system (2.18) are as follows

$$
\begin{aligned}
& x_{2 n-1}=\frac{\left(a y_{0}+b\right) G_{2 n-1}+(b c-a d) y_{0} G_{2 n-2}}{G_{2 n}+\left(c y_{0}-a\right) G_{2 n-1}}, x_{2 n}=\frac{\left(a x_{0}+b\right) G_{2 n}+(b c-a d) x_{0} G_{2 n-1}}{G_{2 n+1}+\left(c x_{0}-a\right) G_{2 n}}, \\
& y_{2 n-1}=\frac{\left(a x_{0}+b\right) G_{2 n-1}+(b c-a d) x_{0} G_{2 n-2}}{G_{2 n}+\left(c x_{0}-a\right) G_{2 n-1}}, y_{2 n}=\frac{\left(a y_{0}+b\right) G_{2 n}+(b c-a d) y_{0} G_{2 n-1}}{G_{2 n+1}+\left(c y_{0}-a\right) G_{2 n}},
\end{aligned}
$$

where $G_{n}$ is a generalized Fibonacci sequence defined by

$$
G_{n+2}=(a+d) G_{n+1}+(b c-a d) G_{n},
$$

with $G_{0}=0$ and $G_{1}=1$. Moreover, they presented that every solution of system (2.18) converges to its equilibrium points.

In [17], Öcalan and Duman considered the following nonlinear recursive difference equation

$$
x_{n+1}=\frac{x_{n-1}}{x_{n}}, n=0,1, \ldots,
$$

with any nonzero initial values $x_{-1}$ and $x_{0}$. Then, they extended their all results to solutions of the following nonlinear recursive equations

$$
x_{n+1}=\left(\frac{x_{n-1}}{x_{n}}\right)^{p}, p>0 \text { and } n=0,1, \ldots,
$$

with any nonzero initial values $x_{-1}$ and $x_{0}$. Later, they obtained that the exact solution of Eq. (2.19) is

$$
x_{n}= \begin{cases}\frac{x_{-1}^{f_{n-1}}}{x_{0}^{f_{n}}} & \text { if } n=1,3,5, \ldots, \\ \frac{x_{0}^{f_{n}}}{x_{-1}^{f_{n-1}}} & \text { if } n=2,4,6, \ldots,\end{cases}
$$

where $f_{n}$ is the $n$th Fibonacci number. Under the special case of initial values, they determined that there exist non-oscillatory positive solutions of Eq. (2.19), which converge monotonically to the equilibrium point 1.

Furthermore, they given that the exact solution of Eq. (2.20) is

$$
x_{n}= \begin{cases}\frac{x_{-1}^{g_{n-1}(p)}}{x_{0}^{f_{n}(p)}} & \text { if } n=1,3,5, \ldots, \\ \frac{x_{0}^{f_{n}(p)}}{x_{-1}^{f_{n-1}(p)}} & \text { if } n=2,4,6, \ldots,\end{cases}
$$

where $f_{n}(p)$ and $g_{n}(p)$ are the $n$th Fibonacci-type number. And also, under the special case of initial values, they demonstrated that there exist non-oscillatory positive solutions of Eq. (2.20), which converge monotonically to the equilibrium point 1 and the Eq. (2.20) has unbounded solutions. 
Next, Akrour et al. [18] studied the following system of difference equations

$$
x_{n+1}=\frac{a y_{n} x_{n-1}+b x_{n-1}+c}{y_{n} x_{n-1}}, y_{n+1}=\frac{a x_{n} y_{n-1}+b y_{n-1}+c}{x_{n} y_{n-1}}, n=0,1, \ldots,
$$

where the parameters $a, b, c$ are arbitrary real numbers with $c \neq 0$ and the initial values $x_{-1}, x_{0}, y_{-1}$ and $y_{0}$ are arbitrary nonzero real numbers. They examined that the explicit solutions of system (2.10) are given by

$$
\begin{aligned}
x_{2 n+1}= & \frac{c J_{2 n+1}+\left(J_{2 n+3}-a J_{2 n+2}\right) x_{-1}+J_{2 n+2} x_{-1} y_{0}}{c J_{2 n}+\left(J_{2 n+2}-a J_{2 n+1}\right) x_{-1}+J_{2 n+1} x_{-1} y_{0}}, \\
x_{2 n+2} & =\frac{c J_{2 n+2}+\left(J_{2 n+4}-a J_{2 n+3}\right) y_{-1}+J_{2 n+3} x_{0} y_{-1}}{c J_{2 n+1}+\left(J_{2 n+3}-a J_{2 n+2}\right) y_{-1}+J_{2 n+2} x_{0} y_{-1}}, \\
y_{2 n+1} & =\frac{c J_{2 n+1}+\left(J_{2 n+3}-a J_{2 n+2}\right) y_{-1}+J_{2 n+2} x_{0} y_{-1}}{c J_{2 n}+\left(J_{2 n+2}-a J_{2 n+1}\right) y_{-1}+J_{2 n+1} x_{0} y_{-1}} \\
y_{2 n+2} & =\frac{c J_{2 n+2}+\left(J_{2 n+4}-a J_{2 n+3}\right) x_{-1}+J_{2 n+3} x_{-1} y_{0}}{c J_{2 n+1}+\left(J_{2 n+3}-a J_{2 n+2}\right) x_{-1}+J_{2 n+2} x_{-1} y_{0}},
\end{aligned}
$$

where $J_{n}$ is defined by the recurrent relation

$$
J_{n+3}=a J_{n+2}+b J_{n+1}+c J_{n}, n \in \mathbb{N},
$$

such that $J_{0}=0, J_{1}=1, J_{2}=a$.

Then, Okumuş and Soykan in [19] considered the following four difference equations

$$
\begin{aligned}
& x_{n+1}=\frac{1}{x_{n}\left(x_{n-1}-1\right)-1}, \quad n=0,1, \ldots, \\
& x_{n+1}=\frac{1}{x_{n}\left(x_{n-1}+1\right)+1}, \quad n=0,1, \ldots, \\
& x_{n+1}=\frac{-1}{x_{n}\left(x_{n-1}-1\right)+1}, \quad n=0,1, \ldots, \\
& x_{n+1}=\frac{-1}{x_{n}\left(x_{n-1}+1\right)-1}, \quad n=0,1, \ldots,
\end{aligned}
$$

and determined the solutions of these difference equations are associated to Tribonacci numbers. For example, the solutions of Eq.(2.21) are

$$
x_{n}=\frac{T_{n-1} x_{-1} x_{0}+\left(T_{n+1}-T_{n}\right) x_{0}+T_{n}}{T_{n} x_{-1} x_{0}+\left(T_{n-1}+T_{n}\right) x_{0}+T_{n+1}},
$$

where the initial conditions $x_{-1}, x_{0} \in \mathbb{R}-F_{2}$, with $F_{2}$ is the forbidden set of Eq.(2.21) given by

$$
F_{2}=\cup_{n=-1}^{\infty}\left\{\left(x_{-1}, x_{0}\right): T_{n} x_{-1} x_{0}+\left(T_{n-1}+T_{n}\right) x_{0}+T_{n+1}=0\right\},
$$

and for the others see [19].

Also, in [21], they examined the following systems of difference equations

$$
x_{n+1}=\frac{ \pm 1}{y_{n}\left(x_{n-1} \pm 1\right)+1}, \quad y_{n+1}=\frac{ \pm 1}{x_{n}\left(y_{n-1} \pm 1\right)+1}, n=0,1, \ldots,
$$

and proved the exact solutions of these systems of difference equations via Tribonacci numbers. E.g. the form of solutions $\left\{x_{n}, y_{n}\right\}_{n=-1}^{\infty}$ of one of these systems is given by

$$
\begin{aligned}
x_{2 n-1} & =\frac{T_{2 n-2} x_{-1} y_{0}+\left(T_{2 n}-T_{2 n-1}\right) y_{0}+T_{2 n-1}}{T_{2 n-1} x_{-1} y_{0}+\left(T_{2 n-2}+T_{2 n-1}\right) y_{0}+T_{2 n}}, \\
x_{2 n} & =\frac{T_{2 n-1} y_{-1} x_{0}+\left(T_{2 n+1}-T_{2 n}\right) x_{0}+T_{2 n}}{T_{2 n} y_{-1} x_{0}+\left(T_{2 n-1}+T_{2 n}\right) x_{0}+T_{2 n+1}}, \\
y_{2 n-1} & =\frac{T_{2 n-2} y_{-1} x_{0}+\left(T_{2 n}-T_{2 n-1}\right) x_{0}+T_{2 n-1}}{T_{2 n-1} y_{-1} x_{0}+\left(T_{2 n-2}+T_{2 n-1}\right) x_{0}+T_{2 n}}, \\
y_{2 n} & =\frac{T_{2 n-1} x_{-1} y_{0}+\left(T_{2 n+1}-T_{2 n}\right) y_{0}+T_{2 n}}{T_{2 n} x_{-1} y_{0}+\left(T_{2 n-1}+T_{2 n}\right) y_{0}+T_{2 n+1}},
\end{aligned}
$$


where the initial conditions $x_{-1}, y_{-1}, x_{0}, y_{0} \in \mathbb{R}-F_{1}$, with $F_{1}$ is the forbidden set of system given by

$$
F_{1}=\cup_{n=-1}^{\infty}\left\{\left(x_{-1}, y_{-1}, x_{0}, y_{0}\right): A_{n}=0 \text { or } B_{n}=0 \text { or } C_{n}=0 \text { or } D_{n}=0\right\}
$$

where

$$
\begin{aligned}
A_{n} & =T_{2 n-1} x_{-1} y_{0}+\left(T_{2 n-2}+T_{2 n-1}\right) y_{0}+T_{2 n}, \\
B_{n} & =T_{2 n} y_{-1} x_{0}+\left(T_{2 n-1}+T_{2 n}\right) x_{0}+T_{2 n+1}, \\
C_{n} & =T_{2 n-1} y_{-1} x_{0}+\left(T_{2 n-2}+T_{2 n-1}\right) x_{0}+T_{2 n}, \\
D_{n} & =T_{2 n} x_{-1} y_{0}+\left(T_{2 n-1}+T_{2 n}\right) y_{0}+T_{2 n+1} .
\end{aligned}
$$

Next, they in [22] studied the following difference equation

$$
x_{n+1}=\frac{\gamma}{x_{n}\left(x_{n-1}+\alpha\right)+\beta}, \quad n=0,1, \ldots
$$

where the parameters $\alpha, \beta$ and $\gamma$ are nonnegative real numbers with $\gamma \neq 0$ and the initial values $x_{-1}$ and $x_{0}$ are arbitrary nonzero real numbers. They examined that the exact solutions of Eq.(2.22) is given by

$$
x_{n}=\frac{t V_{n-1} x_{-1} x_{0}+\left(V_{n+1}-r V_{n}\right) x_{0}+V_{n}}{t V_{n} x_{-1} x_{0}+\left(V_{n+2}-r V_{n+1}\right) x_{0}+V_{n+1}},
$$

where $V_{n}$ is defined by the recurrent relation

$$
V_{n+3}=r V_{n+2}+s V_{n+1}+t V_{n}, n \in \mathbb{N},
$$

such that $V_{0}=0, V_{1}=1, V_{2}=r$.

Besides these studies, for related studies on solving difference equations and systems of difference equations and investigating the asymptotic behavior of their solutions, see [20, 23-38].

\section{References}

[1] D.T. Tollu, Y. Yazlik, N. Taskara, On the solutions of two special types of Riccati difference equation via Fibonacci numbers, Adv. Differ. Equ., 2013 (2013), 174.

[2] J. F. T. Rabago, On the closed-form solution of a nonlinear difference equation and another proof to Sroysang's conjecture, arXiv:1604.06659v1 [math.NT] (2016).

[3] Y. Yazlik, D. T. Tollu, N. Taskara, On the solutions of difference equation systems with Padovan numbers, Appl. Math., 4 (2013), 15-20.

[4] D. T. Tollu, Y. Yazlik, N. Taskara, The solutions of four Riccati difference equations associated with Fibonacci numbers, Balkan J. Math., 2 (2014), 163-172.

[5] D. T. Tollu, Y. Yazlik, N. Taskara, On fourteen solvable systems of difference equations, Appl. Math. Comput., 233 (2014), 310-319.

[6] Y. Halim, Global character of systems of rational difference equations, Elect. J. Mathe. Anal. Appl., 3(1) (2015), $204-214$.

[7] J.B. Bacani, J. F. T. Rabago, On two nonlinear difference equations. Dynamics of continuous, Discrete Impul. Sys., (Serias A) to appear (2015).

[8] Y. Halim, M. Bayram, On the solutions of a higher-order difference equation in terms of generalized Fibonacci sequences, Mathe. Meth. Appl. Sci., 39 (2016), 2974-2982.

[9] Y. Halim, A System of difference equations with solutions associated to Fibonacci numbers, Int. J. Differ Equ., 11(1) (2016), 65-77.

[10] M. M. El-Dessoky, On the dynamics of higher order difference equations $x_{n+1}=a x_{n}+\frac{\alpha x_{n} x_{n-l}}{\beta x_{n}+\gamma x_{n-k}}$, J. Comput. Anal. Appl., 22(7) (2017), 1309-1322.

[11] Y. Halim, J. F. T. Rabago, On some solvable systems of difference equations with solutions associated to Fibonacci numbers, Elect. J. Mathe. Anal. Appl., 5(1) (2017), 166-178. 
[12] Y. Halim, J. F. T. Rabago, On the solutions of a second-order difference equation in terms of generalized Padovan sequences, Math. Slovaca, 68(3) (2018), 625-638.

[13] S. Stevic, B. Iricanin, W. Kosmala, Z. Smarda, Representation of solutions of a solvable nonlinear difference equation of second order, Elect. J. Qualitative Theory of Dif. Equ., 95 (2018), 1-18.

[14] A. M. Alotaibi, M. S. M. Noorani, M. A. El-Moneam, On the solutions of a system of third-order rational difference equations, Discrete Dyn. Nat. Soc., 2018 (2018), Article ID 1743540, 11 pages.

[15] M. M. El-Dessoky, E. M. Elabbasy, A. Asiri, Dynamics and solutions of a fifth-order nonlinear difference equations, Discrete Dyn. Nat. Soc., 2018 (2018), Article ID 9129354, 21 pages.

[16] H. Matsunaga, R. Suzuki, Classification of global behavior of a system of rational difference equations, Appl. Math. Lett., 85 (2018), 57-63.

[17] Ö. Öcalan, O. Duman, On solutions of the recursive equations $x_{n+1}=x_{n-1}^{p} / x_{n}^{p}(p>0)$ via Fibonacci-type sequences, Elect. J. Math. Anal. Appl., 7(1) (2019), 102-115.

[18] Y. Akrour, N. Touafek, N, Y. Halim, On a system of difference equations of second order solved in a closed form, arXiv:1904.04476v1, [math.DS] (2019).

[19] İ. Okumuş, Y. Soykan, On the solutions of four rational difference equations associated to Tribonacci numbers, Preprints, (2019), preprints201906.0266.v1

[20] İ. Okumuş, Y. Soykan, On the solutions of four second-order nonlinear difference equations, Preprints, (2019), preprints201906.0265.v1.

[21] İ. Okumuş, Y. Soykan, On the solutions of systems of difference equations via Tribonacci numbers, arXiv preprint, (2019), arXiv:1906.09987v1 [math.DS].

[22] İ. Okumuş, Y. Soykan, On the dynamics of solutions of a rational difference equation via generalized Tribonacci numbers, arXiv preprint, (2019), arXiv:1906.11629v1 [math.DS].

[23] A. Asiri, E. M. Elsayed, Dynamics and solutions of some recursive sequences of higher order, J. Comput. Anal. Appl., 27(4) (2019), 656-670.

[24] A. Raouf, Global behaviour of the rational Riccati difference equation of order two: the general case, J. Differ. Equ. Appl., 18(6) (2012), 947-961.

[25] E. M. Elsayed, Solution for systems of difference equations of rational form of order two, Comput. Appl. Math., 33 (2014), 751-765.

[26] E. M. Elsayed, Periodicity and solutions for some systems of nonlinear rational difference equations, Hacettepe J. Math. Sta., 44(6) (2015), 1361-1390.

[27] M. Dehghan, R. Mazrooei-Sebdani, H. Sedaghat, Global behaviour of the Riccati difference equation of order two, J. Differ. Equ. Appl., 17(4) (2011), 467-477.

[28] M. Göcen, A. Cebeci, On the periodic solutions of some systems of higher order difference equations, Rocky Mountain J. Math., 48(3) (2018), 845-858.

[29] M. Göcen, M. Güneysu, The global attractivity of some rational difference equations, J. Comput. Anal. Appl., 25(7) (2018), 1233-1243.

[30] Y. Halim, N. Touafek, Y. Yazlik, Dynamics behavior of a second-order nonlinear rational difference equation, Turkish J. Math., 39 (2015), 1004-1018.

[31] J. F. T. Rabago, Effective methods on determining the periodicity and form of solutions of some systems of nonlinear difference equations, arXiv preprints, (2015), arXiv:1512.01605v1 [math.DS].

[32] E. Taşdemir, Y. Soykan, Stability of negative equilibrium of a non-linear difference equation, J. Math. Sci. Adv. Appl., 49(1) (2018), 51-57.

[33] E. Taşdemir, Y. Soykan, Dynamical analysis of a non-linear difference equation, J. Comput. Anal. Appl., 26(2) (2019), 288-301.

[34] S. Stevic, More on a rational recurrence relation, Appl. Math. E-Notes, 4 (2004), 80-84.

[35] S. Stevic, On some solvable systems of difference equations, Appl. Math. Comput., 218 (2012), 5010-5018.

[36] S. Stevic, Representation of solutions of bilinear difference equations in terms of generalized Fibonacci sequences, Elect. J. Qualitative Theory Dif. Equ., 67 (2014), 1-15. 
[37] D. T. Tollu, Y. Yazlik, N. Taskara, On a solvable nonlinear difference equation of higher order, Turkish J. Math, 42 (2018), 1765-1778.

[38] B. Yılmazyıldırım, D. T. Tollu, Explicit solutions of a three-dimensional system of nonlinear difference equations, Hittite J. Sci. Eng., 5(2) (2018), 119-123. 